\title{
Availability of outpatient rehabilitation facilities in 2015 in Iran: A nationwide study
}

\author{
Nahal Ahmadzadeh ${ }^{1}$, Farbod Ebadi Fard Azar*1, Hamid Reza Baradaran², Reza Salman Roghani ${ }^{3}$, \\ Marzieh Shirazikhah ${ }^{4}$, Mohammad Taghi Joghataei ${ }^{5}$, Saiedeh Bahrampouri ${ }^{6}$
}

\begin{abstract}
Background: Disability can involve many by diseases, injuries, or aging, and thus access to rehabilitation services has a key role in improving these patients' social life. Since 2008, Iran has improved the access to rehabilitation facilities along with the global convention. Yet, nationwide studies are required to evaluate the fair distribution of centres in each province. Thus, the present study aimed to evaluate the distribution of outpatient rehabilitation services in 32 provinces of Iran to help policymakers make more appropriate decisions.
\end{abstract}

Methods: In this cross sectional study, which was conducted between April and October 2017, a master facilities list (MFL) of rehabilitation services that was developed in Iran was used to identify health facilities. Data were collected from the statistics of the Deputy for Rehabilitation of Social Welfare Organization offices and Red Crescent Organization in 32 provinces of Iran. Descriptive data were analysed by SPSS version 22 and reported by percentage and number in 1000000 population; distribution was drawn on Iran's map by the GIS software.

Results: According to the MFL, outpatient rehabilitation services include physical medicine \& rehabilitation, physiotherapy, occupational therapy, speech therapy, audiometry, and orthotics \& prosthetics $(90 \%)$; other rehabilitation centres include general rehabilitation centres, day care centres, and vocational centres $(10 \%)$. The largest number of occupational therapy, physiotherapy, and audiology offices were found in Tehran, where general rehabilitation centres, day care centres, and vocational centres were less than 3 per 1000000 population. There were no rehabilitation centers in 14 provinces, and there were very few physical medicine and rehabilitation centres in most of the provinces.

Conclusion: There was significant difference in the distribution of outpatient rehabilitation facilities in different provinces of Iran and some provinces required urgent attention of policymakers, as they had the least number or no rehabilitation facilities.

Keywords: Iran, Availability, Rehabilitation center, Outpatient facilities

Conflicts of Interest: None declared

Funding: Iran University of Medical Sciences

*This work has been published under CC BY-NC-SA 1.0 license.

Copyright $₫$ Iran University of Medical Sciences

Cite this article as: Ahmadzadeh N, Ebadi Fard Azar F, Baradaran HR, Roghani RS, Shirazikhah M, Joghataei MT, Bahrampouri S. Availability of outpatient rehabilitation facilities in 2015 in Iran: A nationwide study. Med J Islam Repub Iran. 2018 (10 Dec);32:123. https://doi.org/10.14196/mjiri.32.123

\section{Introduction}

Disability is the umbrella term for impairments, activity limitations, and participation restrictions (1). The worldwide prevalence of different categories of disability was

Corresponding author: Dr Farbod Ebadi Fard Azar, ebadifardazar.f@iums.ac.ir

1. Health Management and Economics Research Center, Iran University of Medical Sciences, Tehran, Iran

2. Department of Epidemiology, School of Public Health, Iran University of Medical Sciences, Tehran, Iran

3. Physical Medicine \& Rehabilitation Research Center, Shahid Beheshti University of Medical Sciences, Tehran, Iran

4. Social Determinants of Health Research Center, University of Social Welfare and Rehabilitation Sciences, Tehran, Iran

5. Department of Anatomy and Neuroscience, Cellular and Molecular Research Center, School of Medicine, Iran University of Medical Sciences, Tehran, Iran

6. Health in Emergency and Disaster Research Center, University of Social Welfare \& Rehabilitation Sciences, Tehran, Iran reported to be one billion among the world's population ( $15 \%$ of the world's population) by the world health organization (WHO) in 2011 (2).

\section{$\uparrow$ What is "already known" in this topic:}

Universal coverage of rehabilitation services is important for disabled persons; and availability of outpatient rehabilitation facilities is one of the main indicators for assessment of rehabilitation coverage.

\section{$\rightarrow$ What this article adds:}

This study depicts the availability of rehabilitation facilities at the outpatient level and shows geographic distribution of outpatient rehabilitation facilities in different organizations in the country. Evidence was developed to adjust, plan, and decide on the extent and distribution of new rehabilitation centres. 
However, despite these facts, rehabilitation is not considered as a priority to facilitate resources allocation; therefore, there is little funding for rehabilitation in Iran.

The estimated prevalence of disability in Iran was 13 per 10000 population that increases with age (3). The increasing prevalence of chronic diseases, population aging, road accidents, natural disasters, war and climate changes add to the importance of disability in Iran (4). Thus, many people may experience disability temporarily or permanently in their lifetime. However, there is not enough resources to provide rehabilitation services (4), which highlights the importance of access to rehabilitation services that has recently attracted the attention of policymakers in this field.

Since 2008, a total of 152 countries, including Iran, have accepted the United Nations' Convention on the Rights of People with Disabilities (CRPD) that emphasizes universal health coverage and equal access to health services for all people with disabilities and requires governments to plan for sufficient rehabilitation services. One of the most important issues currently discussed in Iran is universal rehabilitation services coverage (5), which ensures that all people can use the required rehabilitative services that they need and ensures all kinds of services are available and accessible to all those who need them (6).

One of the most important indicators for achieving universal health services coverage is availability of services (7), which refers to the physical presence of the rehabilitation services with appropriate regional distribution (8) that affects the health consequences of people with disability (9). Rehabilitation services availability is different among communities (10). According to the 2013 Health and Population Report in the USA, there are about 14.9 days rehabilitation centres per 1000000 population and the number of home care centres is 37.9 and 11.5 respite centres per 1000000 population (11) .In Iran, outpatient rehabilitation services include general rehabilitation centres, physiotherapy, physical medicine and rehabilitation specialists, occupational therapy, speech therapy, orthotics and prosthetics, audiology office, vocational training, and day rehabilitation centres. In a study, Shirazikhah et al found that 3.406 per 1000000 people refer to rehabilitation facilities in outpatient settings and that they are in the form of multidisciplinary day centres; they also found that there are 1.144 general rehabilitation centres for every 1 000000 population (4). Although this study provided good information about outpatient services in the country, there is still no specific information on how these services are distributed throughout the country. In line with
WHO's recommendations to conduct research and generate evidence about the barriers of access to rehabilitation services, the present study aimed to evaluate the distribution of rehabilitation services in Iran to provide necessary evidence in this regard and to help the policymakers to make appropriate decisions to improve availability of rehabilitation services in Iran.

\section{Methods}

\section{Study design}

In this cross sectional study, which was conducted between April and October 2017, a master facilities list (MFL) of rehabilitation services, developed in Iran, was used to identify the health facilities (4). This list includes all public and private sectors, and non-governmental organizations (NGOs) in outpatient settings (Table 1). Three research assistants collected data from the statistics provided by the Deputy for Rehabilitation of Social Welfare Organization offices and the Deputy for Treatment of the Red Crescent Organization in 32 provinces of Iran. The MLF was mailed to 42 medical universities affiliated to the Ministry of Health, and 2 members of the dissertation committee checked the data for accuracy, completeness, and internal consistency.

The sample included all active public and private outpatient physical rehabilitation services affiliated to the Ministry of Health and Medical Education, Ministry of Welfare and Social Security, and the Iranian Red Crescent Society at different settings in 32 provinces in Iran.

\section{Statistical analysis}

National data were analysed for the number of rehabilitation services (public and private centres); nominal and ordinal data were reported by basic descriptive statistics (percentage and frequency); quantitative data were reported by percentage, number in 1000000 people; and distribution was drawn on Iran's map by the GIS software. Data were analysed by SPSS version 22 .

\section{Results}

According to the MFL, availability of outpatient rehabilitation services was assessed based on types of offices (physical medicine \& rehabilitation, physiotherapy, occupational therapy, speech therapy, audiometry, and orthotics \& prosthetics) and other rehabilitation centres that provided team work (general rehabilitation centres, day care centres, and vocational centres). As demonstrated in Figure 1, rehabilitation offices and physiotherapy offices had the most number of outpatient facilities in Iran (29.6:1

Table 1. Infrastructures of master facilities list for outpatient physical rehabilitation services in Iran

\begin{tabular}{ll}
\hline Domain & Tracer indicators, items or services \\
\hline Outpatient & Number of rehabilitation centres per 1000000 population \\
& Number of Physical Medicine \& Rehabilitation offices per 1000000 population \\
& Number of Physiotherapy offices per 1000000 population \\
& Number of Occupational therapy offices per 1000000 population \\
& Number of Speech therapy offices per 1000000 population \\
& Number of Audiology offices per 1000000 population \\
& Number of Orthotic \& Prosthetic offices per 1000000 population \\
& Number of Day care centres per 1000000 population \\
& Number of Vocational centres per 1000000 population \\
\hline
\end{tabular}




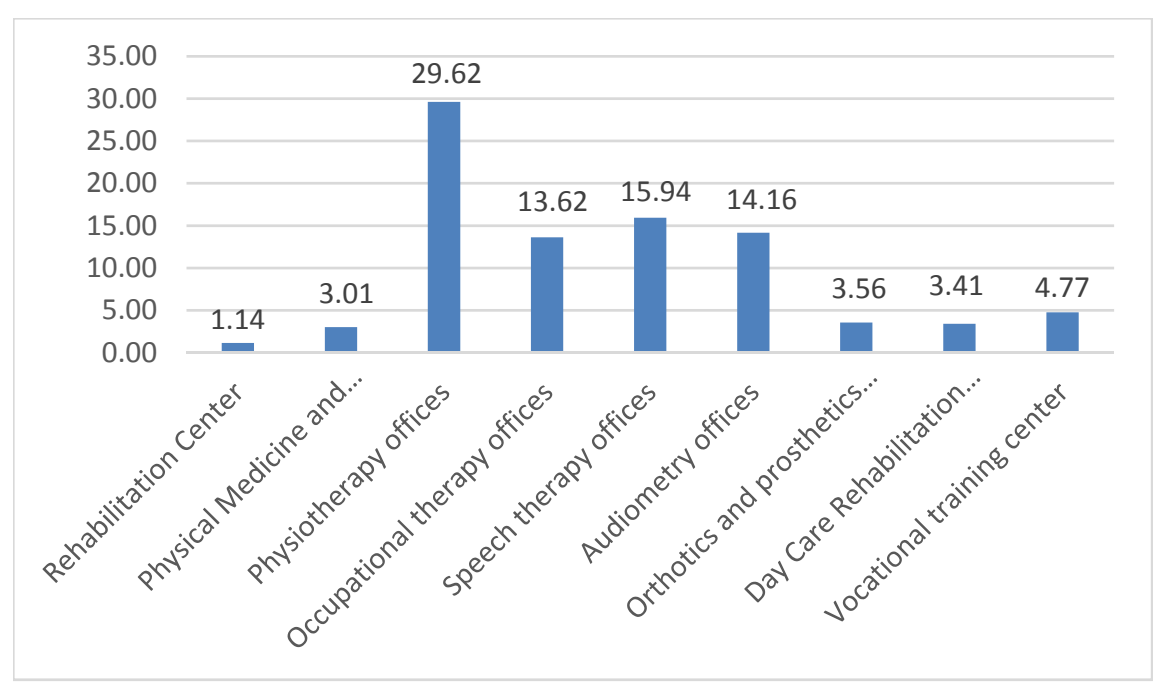

Fig. 1. The total number and frequency of outpatient rehabilitation facilities in Iran

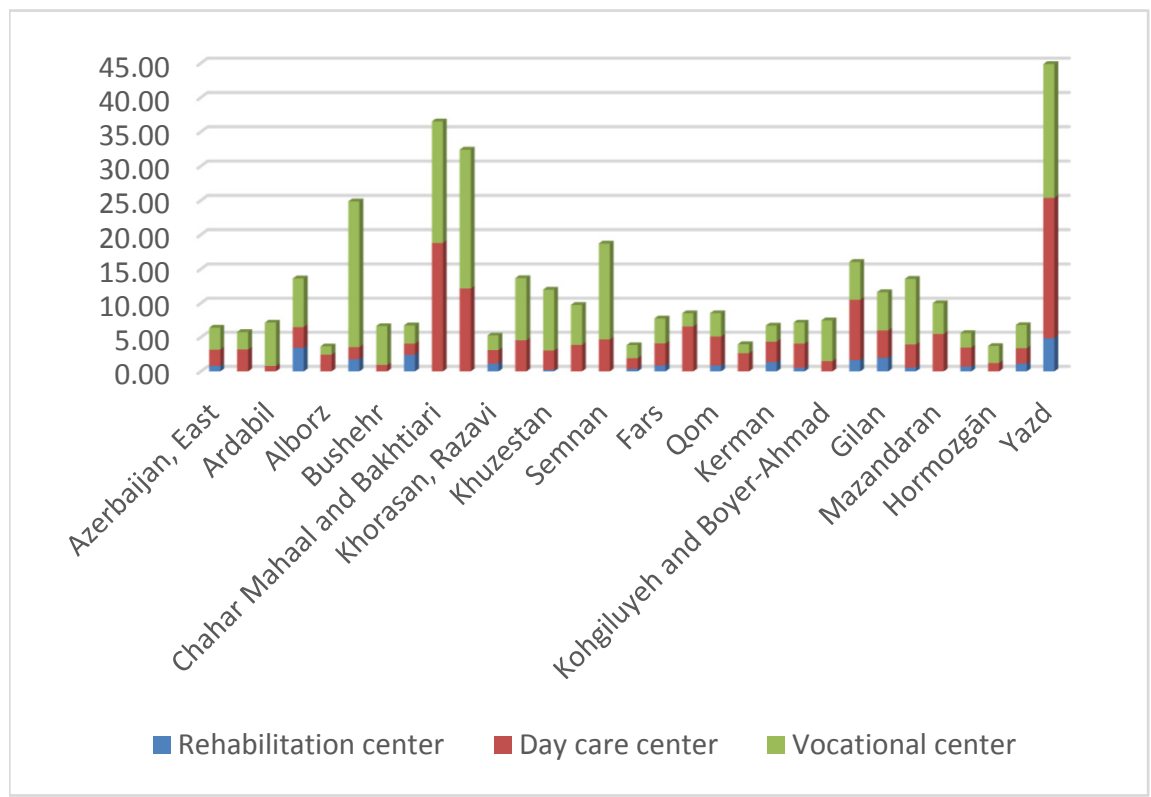

Fig. 2. The distribution of rehabilitation centres, day care centres, and vocational centres based on each province in Iran

$000000)$, followed by physical medicine \& rehabilitation $(0.4 \%)$, physiotherapy $(33 \%)$, occupational therapy $(15 \%)$, speech therapy $(18 \%)$, audiology $(16 \%)$, and orthotics \& prosthetics (4\%), which altogether included $90 \%$ of the total outpatient facilities. Rehabilitation centres $(1 \%)$, day care centres $(4 \%)$, and vocational centres $(5 \%)$ included the rest $(10 \%)$ of the outpatient facilities (Fig. 2).

In the next step, availability of the centres was calculated according to each province. Based on the results, the greatest number of physical medicine \& rehabilitation offices were in Fars province (7.7:1 000 000), but there was none in ChaharMahal and Bakhtiari, Sistan and Baluchistan, and Hormozgan provinces. The highest number of occupational therapy offices existed in Tehran $(35.5: 1$ 000 000) and the lowest in Hormozgan province $(0.6: 1$ $000000)$. The maximum number of speech therapy offices were in Semnan (31.2:1 000 000) and the minimum in Qazvin province $(4.2: 1000000)$. The most and least physiotherapy offices were in Tehran (104.32:1 000 000) and in North Khorasan (14.8:1 000 000), respectively. Also, the largest number of audiology offices were located in Tehran (33.39:1 000 000) and the least in Sistan and Baluchistan province $(3.5: 1000000)$, respectively. The largest and smallest number of orthotic \& prosthetic offices were available in Yazd province (11.7:1 000 000) and Sistan and Baluchistan province $(0.4: 1000000)$, respectively. The number of centers per 1000000 population is demonstrated in Table 2.

Most rehabilitation centres were in Yazd province (4.9:1 000000$)$ and there was none in 14 provinces (Table 2) and the highest and lowest number of day care centres were in Yazd province (20.5:1 000 000) and Ardabil (0.8:1 000 000), respectively. Also, Ilam (21.31:1 000 $000)$ and Alborz provinces (1.2:1 000 000) had the most and the least number of vocational centres, respectively.

To demonstrate the variations in the distribution of comprehensive rehabilitation centres in different provinc- 


\begin{tabular}{|c|c|c|c|c|c|c|c|c|c|}
\hline Province & 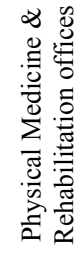 & 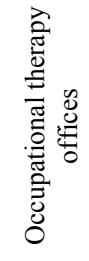 & 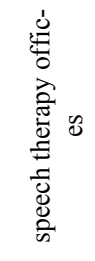 & 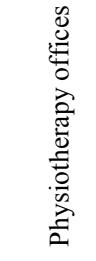 & 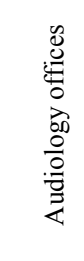 & 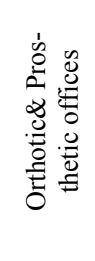 & 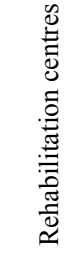 & 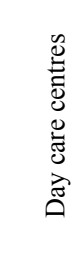 & 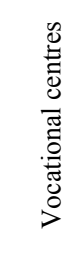 \\
\hline East Azerbaijan & 5.86 & 8.00 & 5.86 & 29.32 & 7.20 & 2.67 & 0.80 & 2.40 & 3.20 \\
\hline West Azerbaijan & 2.89 & 4.81 & 6.09 & 25.00 & 6.41 & 0.64 & 0 & 3.21 & 2.56 \\
\hline Ardabil & 3.18 & 7.95 & 7.15 & 20.67 & 10.33 & 2.39 & 0 & 0.80 & 6.36 \\
\hline Isfahan & 1.83 & 10.37 & 25.19 & 50.39 & 8.76 & 3.66 & 3.45 & 3.05 & 7.11 \\
\hline Alborz & 3.26 & 20.77 & 18.72 & 38.26 & 17.09 & 3.66 & 0 & 2.44 & 1.22 \\
\hline Ilam & 0 & 12.43 & 19.64 & 19.40 & 12.43 & 1.78 & 1.78 & 1.78 & 21.31 \\
\hline Bushehr & 3.79 & 2.84 & 8.86 & 29.38 & 9.48 & 4.74 & 0 & 0.95 & 5.69 \\
\hline Tehran & 6.36 & 35.51 & 28.76 & 104.32 & 33.39 & 7.96 & 2.44 & 1.63 & 2.68 \\
\hline Chaharmahal and Bakhtiari & 0 & 11.06 & 15.49 & 34.29 & 8.85 & 5.53 & 0 & 18.81 & 17.70 \\
\hline South Khorasan & 1.35 & 13.50 & 22.94 & 16.19 & 13.50 & 2.70 & 0 & 12.15 & 20.24 \\
\hline Razavi Khorasan & 1.32 & 11.02 & 16.61 & 34.20 & 11.51 & 1.15 & 1.15 & 1.97 & 2.14 \\
\hline North Khorasan & 0 & 7.97 & 13.67 & 14.81 & 11.39 & 3.42 & 0 & 4.56 & 9.11 \\
\hline Khuzestan & 1.09 & 6.33 & 12.62 & 49.40 & 8.05 & 1.52 & 0.22 & 2.84 & 8.92 \\
\hline Zanjan & 2.92 & 4.87 & 8.77 & 15.59 & 9.75 & 4.87 & 0 & 3.90 & 5.85 \\
\hline Semnan & 4.68 & 29.64 & 31.20 & 74.88 & 18.72 & 4.68 & 0 & 4.68 & 14.04 \\
\hline Sistan and Baluchestan & 0 & 2.31 & 4.62 & 25.04 & 3.47 & 0.39 & 0.39 & 1.54 & 1.93 \\
\hline Fars & 7.76 & 9.91 & 14.86 & 72.38 & 8.18 & 0.65 & 0.86 & 3.23 & 3.66 \\
\hline Qazvin & 0.82 & 8.24 & 4.24 & 28.01 & 9.89 & 4.94 & 0 & 6.59 & 1.93 \\
\hline Qom & 3.41 & 20.48 & 24.74 & 51.20 & 20.48 & 5.12 & 0.85 & 4.27 & 3.41 \\
\hline Kurdistan & 1.33 & 6.65 & 12.63 & 17.95 & 9.31 & 3.99 & 0 & 2.66 & 1.33 \\
\hline Kerman & 1.68 & 10.06 & 15.09 & 32.53 & 15.09 & 1.68 & 1.34 & 3.02 & 2.35 \\
\hline Kermanshah & 0.51 & 8.70 & 9.21 & 28.15 & 11.26 & 5.63 & 0.51 & 3.57 & 3.07 \\
\hline Kohgiluyeh and Boyer-Ahmad & 1.50 & 10.43 & 17.94 & 35.77 & 25.41 & 1.50 & 0 & 1.50 & 5.98 \\
\hline Golestan & 1.66 & 7.74 & 9.96 & 38.72 & 10.51 & 2.21 & 1.66 & 8.85 & 5.53 \\
\hline Gilan & 1.20 & 8.01 & 8.41 & 45.67 & 9.62 & 3.21 & 2.00 & 4.00 & 5.61 \\
\hline Lorestan & 1.70 & 5.09 & 9.04 & 22.60 & 7.91 & 5.09 & 0.57 & 3.39 & 9.61 \\
\hline Mazandaran & 2.90 & 9.03 & 15.81 & 61.61 & 13.23 & 1.29 & 0 & 5.48 & 4.52 \\
\hline Markazi & 0.70 & 17.51 & 13.31 & 32.21 & 20.31 & 2.80 & 0.70 & 2.80 & 2.10 \\
\hline Hormozgan & 0.62 & 0.62 & 4.35 & 14.91 & 6.21 & 0.62 & 0 & 1.24 & 2.48 \\
\hline Hamadan & 2.26 & 12.44 & 9.05 & 27.15 & 14.71 & 6.79 & 1.13 & 2.26 & 3.39 \\
\hline Yazd & 1.95 & 14.63 & 27.32 & 48.78 & 14.63 & 11.71 & 4.88 & 20.49 & 19.51 \\
\hline
\end{tabular}

* Offices include hospital-based outpatient units, health center and private clinics

es of Iran, the number of each center is demonstrated on Iran's map (Fig. 3).

\section{Discussion}

This study was conducted to determine the availability of outpatient rehabilitation facilities in Iran. Different facilities are available in outpatient setting in Iran, including physical medicine \& rehabilitation centres, physiotherapy, occupational therapy, speech therapy, audiometry, orthotic \& prosthetic and general rehabilitation cen- tres (eg, day care centres and vocational centres).

According to the results, the number of facility centres in Iran was high for physiotherapy centres (about 30: 1 000000 ) and moderate for speech therapy, audiology offices, and occupational therapy centres (about 13-16: 1 000 000), while the number of physical rehabilitation offices was very limited in Iran (about 3: 1000 000). In other words, the number of centres that offer comprehensive rehabilitation services was clearly lower than other centres and constituted only the $10 \%$ of the total centres. In Iran, 

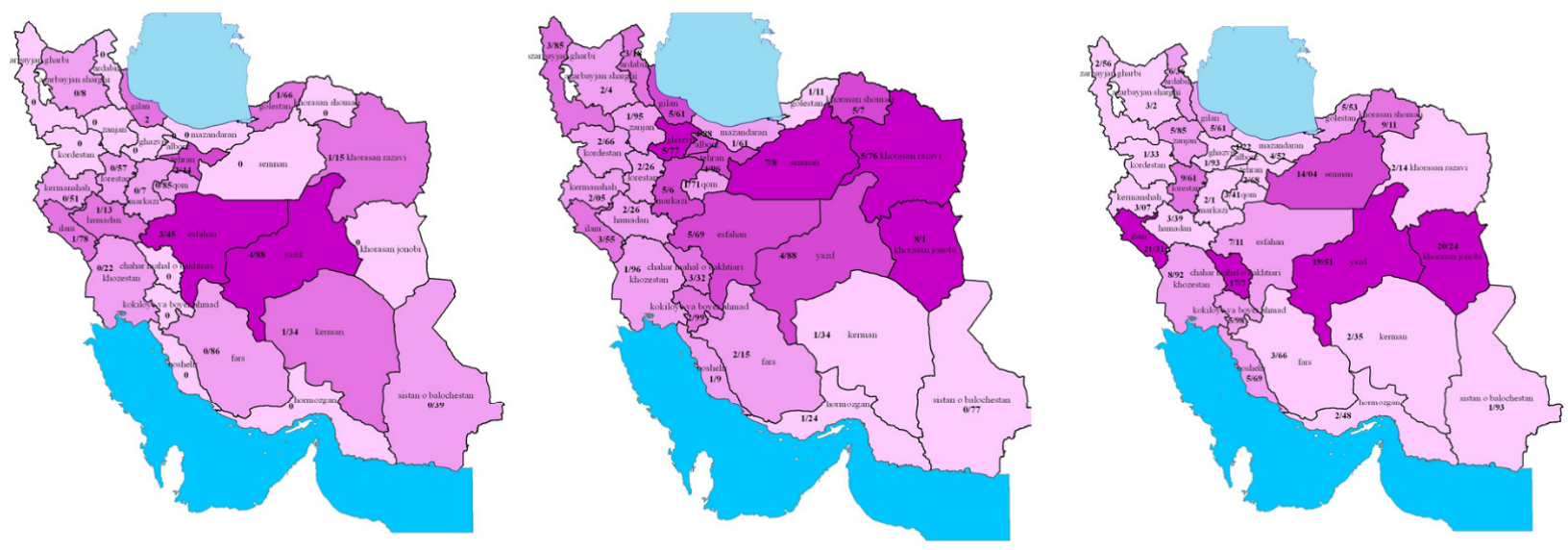
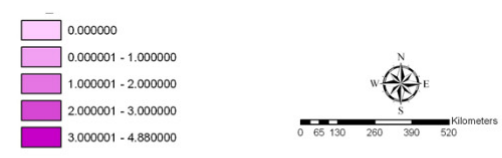

The number of rehabilitation centers in each province
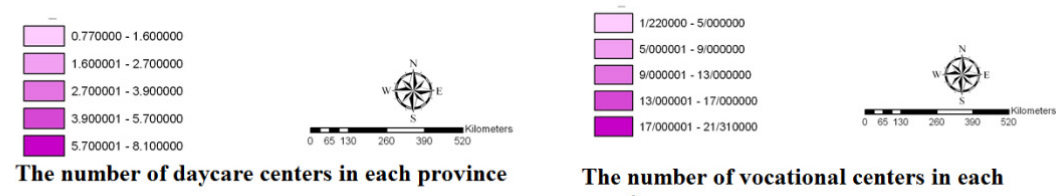

The number of vocational centers in each province

Fig. 3. The number of rehabilitation centres, day care centres, and vocational centres in each province of Iran

rehabilitation services are provided in different settings, including inpatient, outpatient, community-based, and long-term care centres (4). Outpatient rehabilitation services are supervised by the Ministry of Health and Medical Education, the Ministry of Welfare and Social Security, and the Iranian Red Crescent Society (12-14). Lack of integration and information registration system between the service providers could be the main reason for this unfair distribution of different types of facility centres in Iran. This matter has to be considered by policymakers, as patients with disabilities need to use a variety of rehabilitation services and comprehensive rehabilitation can help deliver coordinated rehabilitation services. In addition, having an interdisciplinary rehabilitation team with good management can provide essential affordable services (15), which should be considered more seriously in Iran.

Few Iranian studies have addressed rehabilitation and those who did have mainly focused on a specific group such as the elderly. In a study conducted by Abdi et al in Kashan, the necessity of building capacity for rehabilitation facilities in the health system was emphasized (16). Dalvandi et al conducted a study on patients after stroke and they found that lack of access to rehabilitative services led to life disintegration, functional disorder, and poor socioeconomic state (17). In the study of Farkhondeh Sharif et al, it was found that cardiac rehabilitation was effective in reducing depression 2 months after surgery in patients with coronary artery bypass graft (18) in 1 centre and, to our knowledge, no nationwide study has evaluated the number of rehabilitation centres. A study in Tehran has reported an accessibility of rehabilitation services as a major barrier based on interviews with patients and their family members, policymakers, and NGOs (19).

Although the results of the present study indicated limited number of physical rehabilitation offices in the nation, the situation in Iran does not seem very different from some other countries such as 4 South African coun- tries, with access of only $26 \%-55 \%$ of people to rehabilitation services (2). Also, the global reports of WHO indicates that the need for rehabilitation facilities and services is not globally met, especially in low- and middle-income countries, where the estimates are inaccurate $(10,2)$. The results of the present study revealed that in addition to the inappropriate distribution of rehabilitation centres across the country, the number of centres available per 1000000 population in each province of Iran was different. Moreover, there were inadequate number of rehabilitation offices in some cities and no offices in some other cities. However, there were many physiotherapy, speech therapy, and audiology offices in almost all provinces of the country. Even in Tehran, the capital, the number of rehabilitation, day care and vocational centres was less than 3 per 1000 000 population. In fact, rehabilitation centres only existed in 14 provinces and were less than 1 per 1000000 population in these provinces. Also, there were no physical medicine \& rehabilitation offices in Ilam, Chaharmahal and Bakhtiari, North Khorasan, and Sistan and Baluchistan provinces, and there were less than 3 per 1000000 in 17 provinces, indicating insufficient number of physical medicine \& rehabilitation offices in most provinces of Iran.

It was expectable that some deprived provinces, such as Sistan and Baluchistan, have a low number of most rehabilitation offices, but even in that province, the number of physiotherapy centres was more than 25 per 1000000 population. In ChaharMahal and Bakhtiari province, despite the absence or insufficient number of different rehabilitation centres, the number of day care centres and vocational centres was rather high (about 18:1 000 000). Overall, distribution of centres per province indicates that there is no province with appropriate distribution of rehabilitation services, as the number of some types of facility centres was rather small or very large in the provinces. For instance, Yazd had the highest number of rehabilita- 
tion and day care centres in the nation, while the number of physical medicine $\&$ rehabilitation offices was less than 2 per 1000000 in the country. These results indicate the urgent need for managing Iran's rehabilitation services in all provinces. The major limitations of this study could be the retrospective evaluation of the variables based on the sources used for data collection and any bias in the original data.

\section{Conclusion}

This study was the first nationwide study to assess the availability of the rehabilitation services in Iran and was based on the statistics of the Deputy for Rehabilitation of Social Welfare Organization offices and Red Crescent Organization in 32 provinces of Iran. In this study, it was found that the number of most rehabilitation services is insufficient in some provinces of Iran, while in some other provinces, the distribution is inappropriate and the number of some facilities is unexpectedly high. These results call for the attention of policymakers, executive directors, and therapists to eliminate cultural barriers to provide services to the target community to improve this gap and to create facilities for people with disabilities, with particular attention to appropriate distribution of these centres across the country.

\section{Acknowledgements}

This paper is based on $\mathrm{PhD}$ thesis of Ms Nahal Ahmadzadeh supported by Iran University of Medical Sciences. Authors would like to express their deep gratitude to the following organizations: The Ministry of Health and Medical Education, the Social Welfare and Rehabilitation Organization, the Red Crescent Organization, and the Department of Statistics-Medical System Organization.

\section{Ethical approval}

Ethical approval was granted by the ethics committee of Iran University of Medical Sciences.

\section{Informed consent \\ Not applicable}

\section{Conflict of interests}

The authors declare that they have no competing interests. electrodiagnosis, 2012; Available from: http://eprints.ajaums.ac.ir/ 2513/

6. World Health Organization. What is universal coverage? 2015. Available from: http://www.who.int/health_financing/universal_ coverage definition/en/

7. Tanahashi T. Health service coverage and its evaluation. Bulletin of the World Health Organization. 1978;56(2):295.

8. Evans DB, Hsu J, Boerma T. Universal health coverage and universal access. Bullet World Health Organization. 2013;91(8):546-546A.

9. Martinez J, Pampalon R, Hamel D, Raymond G. Does living in rural communities rather than cities really make a difference in people's health and wellness. 2004. Quebec: INSPQ Google Scholar.

10. Gupta N, Castillo-Laborde C, Landry MD. Health-related rehabilitation services: assessing the global supply of and need for human resources. BMC Health Serv Res. 2011;11(1):276.

11. Harris-Kojetin L, Sengupta M, Park-Lee E, Valverde R. Long-Term Care Services in the United States: 2013 Overview. Vital Health Stat 3. 2013;(37):1-107.

12. Behzisti. State welfare organization 2015; Available from: http://www.behzisti.ir./

13. IRCS. Iranian red crescent society. 2015; Available from: http://217.218.20.182/en./

14. Behdasht. Ministry of Health and Medical Education. 2015; Available from: http://www.behdasht.gov.ir./

15. Fuchs H. Alternative fibreless absorbers-New tools and materials for noise control and acoustic comfort. Acta Acust united Ac. 2001;87(3):414-422.

16. Adib-Hajbaghery M, Aghahoseini S. The evaluation of disability and its related factors among the elderly population in Kashan, Iran. BMC Public Health. 2007;7(1): 261.

17. Dalvandi A, Heikkilä K, Maddah SS, Khankeh HR, Ekman SL. Life experiences after stroke among Iranian stroke survivors. Int Nurs Rev. 2010;57(2):247-53

18. Sharif F, Shoul A, Janati M, Kojuri J, Zare N. The effect of cardiac rehabilitation on anxiety and depression in patients undergoing cardiac bypass graft surgery in Iran. BMC Cardiovasc Disord. 2012;12(1): 40 .

19. Abdi K, Arab M, Rashidian A, Kamali M, Khankeh HR, Farahani FK. Exploring barriers of the health system to rehabilitation services for people with disabilities in Iran: A Qualitative Study. Electron Physician. 2015;7(7):14-76.

\section{References}

1. Bethge M, von Groote P, Giustini A, Gutenbrunner C. The World Report on Disability: a challenge for rehabilitation medicine. Am J Phys Med Rehabil. 2014;93:S4-11.

2. World Health Organization. The World Bank. World report on disability. WHO Library Cataloguing-in-Publication Data. 2011. Available from: http://www.who.int/disabilities/world_report/2011/ report.pdf?ua=1. PMID:26131540.

3. Soltani S, Khosravi B, Salehiniya H. Prevalence of disability in Iran. Iran J Public Health. 2015;44(10):1436-7.

4. Shirazikhah M, Mirabzadeh A, Sajadi H, Joghataei MT, Biglarian A, Mousavi T, et al. National survey of availability of physical rehabilitation services in Iran: A mixed methods study. Electron Physician. 2017;9(11):5778-5784.

5. Rayegani S. Physical medicine and rehabilitation specialist position in the structure of the national health and referral system. 16th annual congress of Iranian society of physical medicine, rehabilitation and 